A C G

publications

Org. Commun. 14:3 (2021) 294-299

organic

communications

\title{
Preparation of new mono- and bis-amide derivatives of L-isoleucine via amidation of carboxyl and amino groups
}

\author{
Murat Sunkur (D) 1 , Sara Aydin (D)1, Tarik Aral (D) 1 , Beşir Dag ${ }^{\text {(D) } 1}$ and \\ Ramazan Erenler (iD 2*
}

\author{
${ }^{I}$ Department of Chemistry, Faculty of Arts and Sciences, Batman University, 72060 Batman, Türkiye \\ ${ }^{2}$ Department of Chemistry, Faculty of Arts and Sciences, Tokat Gaziosmanpasa University, 60250
}

Tokat, Türkiye

(Received July 17, 2021; Revised August 25, 2021 Accepted August 26, 2021)

\begin{abstract}
N, N^{\prime}$-Dicyclohexylcarbodiimide and hydroxybenzotriazole mediated amination of free COOH group of $N$-tert-butyloxyoxycarbonyl-L-isoleucine $\mathbf{1}$ with three aromatic amines gave the corresponding amides 2a-c. Deprotection of $\mathrm{N}$-tert- butyloxyoxycarbonyl groups of $\mathbf{2 a - c}$ with $\mathrm{AcOH}$ yielded the related amine derivatives 3a-c. The amidation of the free $\mathrm{NH}_{2}$ groups of (3a-c) with phthaloyl dichloride yielded the bis-amide products 4a-c. The synthesized compounds were purified by crystallization and their structures were elucidated by spectroscopic methods such as ${ }^{1} \mathrm{H}-\mathrm{HMR},{ }^{13} \mathrm{C}-\mathrm{NMR}$, FTIR, and micro analysis.
\end{abstract}

Keywords: Amides; tetraamides; N-boc-deprotection; coupling reaction; spectroscopy; heterocyclic. (C2021 ACG Publications. All right reserved.

\section{Introduction}

Due to the versatility of amides, they have gained a great importance in synthetic chemistry. Numerous methods have been developed to synthesize the amides for usage in the pharmaceutical and medicinal industries. The most common one is the treatment of carboxylic acid with amine. A large variety of natural products include the amide moiety, moreover, many drug formulations contain the amide groups ${ }^{1}$. Chiral amides consisting of vicinal stereo centres commonly exist in natural products and drug molecules.

The compounds having amide groups synthesised chemically were reported recently that revealed a large variety of biological activity such as anticancer ${ }^{2}$, antimicrobial ${ }^{3}$, antiviral ${ }^{4}$, antibacterial ${ }^{5}$, antituberculosis ${ }^{6}$, fungicidal and insecticidal ${ }^{7}$, pesticide ${ }^{8}$, anti- respiratory syncytial virus ${ }^{9}$. These compounds could be preferable ligands for synthesis of metal complex as well. Amide bonds play a major role in explanation and composition of biological system, representing main chemical bonds that link amino acid building blocks together to give proteins. Amide bonds are not limited to biological systems and indeed exist in a wide variety of molecules, including major marketed drugs. For instance, Atorvastatin, consists of amide bond, prevent the formation of cholesterol ${ }^{10}$, lisinopril, inhibitor of angiotensin converting enzyme ${ }^{11}$, valsartan, barricade the angiotensin II receptor ${ }^{12}$, diltiazem, calcium channel blocker, treatment of hypertension ${ }^{13}$. Amide bonds are typically formed by the treatment of carboxylic acids with amines. The removal of tert-butyl carbamate by TFA/DCM was also reported ${ }^{14,15}$

\footnotetext{
* Corresponding author: E-Mail: rerenler@ gmail.com, Phone: + 90 3562521616-3055; Fax: + 903562521585 
Erenler et al., Org. Commun. (2021) 14:3 294-299

Herein, the diastereoselective syntheses of amides bearing two stereogenic centers in the 2,3 positions with respect to nitrogen atom were achieved with a good yield.

\section{Experimental}

\subsection{General Procedure}

NMR spectra were recorded on a Bruker spectrometer with ${ }^{1} \mathrm{H}$ NMR at $400 \mathrm{MHz},{ }^{13} \mathrm{C}$ NMR at $100 \mathrm{MHz}$. The ${ }^{1} \mathrm{H}$ NMR chemical shifts were measured relative to DMSO-d6 as the internal reference $\left(\mathrm{CDCl}_{3}: \delta 7.26\right.$, DMSO-d6: $\left.\delta 2.5\right)$. TLC was carried out on alumina plates $\left(60 \mathrm{~F}_{254}\right)$. TLC spots were observed under UV light and sprayed with ceric sulfate reagent then heated. IR analysis was executed on Jasco FT/IR47700 spectrometer. Melting points were determined by a Buchi B-540 apparatus and were uncorrected. The solvents and chemicals were supplied from E. Merck (Darmstadt, Germany).

\subsection{General Synthesis of Carbamate Derivatives (2a-c)}

N-tert-butyloxycarbonyl-L-isoleucine $1(12.0 \mathrm{mmol})$, hydroxybenzotriazole (HOBt) (14.4 mmol) were dissolved with $\mathrm{CH}_{2} \mathrm{Cl}_{2}(300 \mathrm{~mL})$ in an ice-bath reaction flask. Aromatic amines (a-c) (12.0 $\mathrm{mmol})$ and $N, N^{\prime}$-Dicyclohexylcarbodiimide (DCC) $(13.2 \mathrm{mmol})$ were added to the reaction flask. The reaction mixture was stirred overnight at room temperature. After the completion of the reaction, hexyl cyclo urea formed was removed by filtration. The chloroform was added to the reaction flask, extracted with $1.0 \mathrm{M} \mathrm{HCl}, 5 \% \mathrm{NaHCO}_{3}$ and distilled water successively then organic phase was separated by separatory funnel, dried with calcium chloride, filtered then solvent was removed by rotary evaporator to yield the product (2a-c).

tert-Butyl [(2R,3S)-3-methyl-1-\{[1-(naphthalen-1-yl)ethyl]amino\}-1-oxopentan-2-yl)]carbamate (2a): (4.20 g, 91\%). Mp: $143-144{ }^{\circ} \mathrm{C}$. Anal. Calcd (\%) for $\mathrm{C}_{23} \mathrm{H}_{32} \mathrm{~N}_{2} \mathrm{O}_{3}: \mathrm{C}, 71.84 ; \mathrm{H}, 8.39$. Found: C, 71.79; H, 8.42. IR: $\left(\mathrm{v} / \mathrm{cm}^{-1}\right) 3317,3253,3060,2967,2931,2878,2850,1683,1639,1521,1449,1390,1365$, 1314, 1292, 1244, 1166, 1124, 1085, 1043, 1015, 998, 917, 866, 797, 775 (Figure S1). ${ }^{1} \mathrm{H}$ NMR (400 MHz, DMSO-d 6 ) $\delta: 8.46(\mathrm{~d}, J=7.7 \mathrm{~Hz}, 1 \mathrm{H}), 8.11(\mathrm{~d}, J=7.9 \mathrm{~Hz}, 1 \mathrm{H}), 7.94(\mathrm{~d}, J=7.4 \mathrm{~Hz}, 1 \mathrm{H}), 7.83(\mathrm{~d}$, $J=8.0 \mathrm{~Hz}, 1 \mathrm{H}), 7.53(\mathrm{~m}, 2 \mathrm{H}), 7.47(\mathrm{t}, J=7.6 \mathrm{~Hz}, 1 \mathrm{H}), 6.58(\mathrm{~d}, J=9.0 \mathrm{~Hz}, 1 \mathrm{H}), 5.75-5.72(\mathrm{~m}, 1 \mathrm{H})$, $3.91(\mathrm{t}, J=8.0 \mathrm{~Hz}, 1 \mathrm{H}), 1.60-1.75(\mathrm{~m}, 2 \mathrm{H}), 1.50(\mathrm{~d}, J=6.7 \mathrm{~Hz}, 3 \mathrm{H}), 1.38(\mathrm{~s}, 9 \mathrm{H}), 1.02-1.14(\mathrm{~m}, 2 \mathrm{H})$, $0.76(\mathrm{~m}, 6 \mathrm{H})$ (Figure $\mathrm{S} 2) .{ }^{13} \mathrm{C}$ NMR (100 MHz, DMSO-d 6 ) 170.9 ( $\mathrm{C}=\mathrm{O}$ amide), $155.8(\mathrm{C}=\mathrm{O}$ carbamate), 140.6, 133.8, 130.8, 129.1, 127.8, 126.6, 126.0, 125.7, 123.6, 122.9, 78.4, 59.2, 44.4, 37.3, 28.6, 24.6, 22.0, 15.9, 11.4 (Figure S3).

tert-Butyl [(2R,3S)-1-\{[4-(tert-butyl)benzyl]amino\}-3-methyl-1-oxopentan-2-yl]carbamate (2b): (3.34 g, 74\%). Mp: 118-122 ${ }^{\circ} \mathrm{C}$. Anal. Calcd (\%) for $\mathrm{C}_{22} \mathrm{H}_{36} \mathrm{~N}_{2} \mathrm{O}_{3}:$ C, 70.21; H, 9.57. Found: C, 70.19; H, 9.63. IR: $\left(\mathrm{v} / \mathrm{cm}^{-1}\right) 3345,3315,3266,2962,2931,2872,1686,1642,1515,1459,1390,1365,1317,1292$, 1244, 1172, 1043, 1018, 915 (Figure S4). ${ }^{1} \mathrm{H}$ NMR (400 MHz, DMSO-d 6 ) $\delta: 8.29(\mathrm{t}, J=5.3 \mathrm{~Hz}, 1 \mathrm{H})$, $7.31(\mathrm{~d}, J=8.1 \mathrm{~Hz}, 2 \mathrm{H}), 7.18(\mathrm{~d}, J=8.0 \mathrm{~Hz}, 2 \mathrm{H}), 6.70(\mathrm{~d}, J=8.9 \mathrm{~Hz}, 1 \mathrm{H}), 4.24(\mathrm{~d}, J=5.7 \mathrm{~Hz}, 2 \mathrm{H})$, $3.83(\mathrm{t}, J=8.3 \mathrm{~Hz}, 1 \mathrm{H}), 1.70(\mathrm{~d}, J=5.9 \mathrm{~Hz}, 1 \mathrm{H}), 1.39(\mathrm{~s}, 9 \mathrm{H}), 1.26(\mathrm{~s}, 9 \mathrm{H}), 1.18(\mathrm{t}, J=7.1 \mathrm{~Hz}, 1 \mathrm{H})$, $1.10(\mathrm{~m}, 1 \mathrm{H}), 0.83-0.79(\mathrm{~m}, 6 \mathrm{H})$ (Figure S5). ${ }^{13} \mathrm{C}$ NMR $\left(100 \mathrm{MHz}, \mathrm{DMSO}-\mathrm{d}_{6}\right) \delta 171.9(\mathrm{C}=\mathrm{O}$ amide), 155.8 ( $\mathrm{C}=\mathrm{O}$ carbamate), 149.6, 136.8, 127.5, 125.3, 78.4, 59.4, 42.2, 36.8, 34.6, 31.6, 28.6, 24.9, 16.0, 11.4 (Figure S6).

tert-Butyl [(2R,3S)-1-\{[4-butylbenzyl]amino\}-3-methyl-1-oxopentan-2-yl]carbamate $(2 \mathrm{c}):(4.07 \mathrm{~g}$, 90\%). Mp: $113-118{ }^{\circ} \mathrm{C}$. Anal. Calcd (\%) for $\mathrm{C}_{21} \mathrm{H}_{34} \mathrm{~N}_{2} \mathrm{O}_{3}: \mathrm{C}, 69.61 .21 ; \mathrm{H}, 9.39$. Found: $\mathrm{C}, 69.50 ; \mathrm{H}$, 9.43. IR: $\left(\mathrm{v} / \mathrm{cm}^{-1}\right) 3343,3312,2959,2952,2872,2853,2372,2352,2321,1686,1661,1596,1516$, 1462, 1412, 1365, 1312, 1284, 1244, 1166, 1115, 1046, 1018, 970, 925 (Figure S7). ${ }^{1} \mathrm{H}$ NMR (400 MHz, DMSO-d $\left._{6}\right) \delta 9.91(\mathrm{~s}, 1 \mathrm{H}), 7.51(\mathrm{~d}, J=8.2 \mathrm{~Hz}, 2 \mathrm{H}), 7.11(\mathrm{~d}, J=8.3 \mathrm{~Hz}, 2 \mathrm{H}), 6.90(\mathrm{~d}, J=8.4 \mathrm{~Hz}, 1 \mathrm{H})$, $3.94(\mathrm{t}, J=8.3 \mathrm{~Hz}, 1 \mathrm{H}), 2.51(\mathrm{t}, J=7.2 \mathrm{~Hz}, 2 \mathrm{H}), 1.51(\mathrm{~m}, 4 \mathrm{H}), 1.38(\mathrm{~s}, 9 \mathrm{H}), 1.28(\mathrm{~m}, 4 \mathrm{H}), 1.20-1.04$ $(\mathrm{m}, 1 \mathrm{H}), 0.90-0.79(\mathrm{~m}, 9 \mathrm{H})$ (Figure S8). ${ }^{13} \mathrm{C}$ NMR (100 MHz, DMSO-d 6 ) $\delta 171.0(\mathrm{C}=\mathrm{O}$ amide), 155.9 
Preparation of carboxyl and amino groups

( $\mathrm{C}=\mathrm{O}$ carbamate), 137.7, 137.0, 128.9, 119.7, 78.4, 59.9, 36.9, 34.7, 33.8, 33.7, 28.7, 25.1, 22.1, 15.8, 14.2, 11.3 (Figure S10).

\subsection{General Synthesis of Pentanamide Derivatives (3a-c)}

Carbamate derivatives $(\mathbf{2 a - c})(6.0 \mathrm{mmol})$ was added in a round bottom reaction flask. AcOH (60 mmol), $\mathrm{CF}_{3} \mathrm{COOH}(60 \mathrm{mmol})$ and $\mathrm{CH}_{2} \mathrm{Cl}_{2}(5.0 \mathrm{~mL})$ were added in a dropping funnel. The solution in dropping funnel was added slowly to the reaction flask for half an hour at $0{ }^{\circ} \mathrm{C}$. After finishing the addition, the reaction mixture was stirred at room temperature overnight under the argon. The reaction mixture was completed after TLC application. $1 \mathrm{M} \mathrm{KOH}$ was added to the reaction mixture to adjust the $\mathrm{pH}$ at 9-10, then the $\mathrm{H}_{2} \mathrm{O}(30 \mathrm{~mL})$ was added to the mixture, extracted with $\mathrm{CH}_{2} \mathrm{Cl}_{2}(3 \times 20 \mathrm{~mL})$, organic phase was separated by separatory funnel, dried $\left(\mathrm{CaCl}_{2}\right)$, filtered and solvent was removed by rotary evaporator to yield the product (3a-c).

(2R,3S)-2-amino-3-methyl-N-[1-(naphthalen-1-yl)ethyl]pentanamide (3a): (1.62 g, \%95). Mp: 95-96 ${ }^{\circ} \mathrm{C}$. Anal. Calcd (\%) for $\mathrm{C}_{18} \mathrm{H}_{24} \mathrm{~N}_{2} \mathrm{O}: \mathrm{C}, 76.02 ; \mathrm{H}, 8.51$. Found: C, $76.10 \mathrm{H}, 8.55$. IR: $\left(\mathrm{v} / \mathrm{cm}^{-1}\right) 3368$, $3303,3048,2967,2925,2872,1614,1533,1452,1378,1337,1218,1258,1239,1219$, 1172, 1118 , 1082, 1037, 1024, 996, 973, 902, 869, 859, 833, 797, 775 (Figure S11). ${ }^{1} \mathrm{H}$ NMR (400 MHz, DMSO$\left.\mathrm{d}_{6}\right) \delta 8.35(\mathrm{~d}, J=8.1 \mathrm{~Hz}, 1 \mathrm{H}), 8.13(\mathrm{~d}, J=8.8 \mathrm{~Hz}, 1 \mathrm{H}), 7.93(\mathrm{dd}, J=8.1,6.1 \mathrm{~Hz}, 1 \mathrm{H}), 7.83(\mathrm{~d}, J=8.0$ $\mathrm{Hz}, 1 \mathrm{H}), 7.57-7.47(\mathrm{~m}, 4 \mathrm{H}), 5.75(\mathrm{~m}, 1 \mathrm{H}), 3.03(\mathrm{~d}, J=5.4 \mathrm{~Hz}, 1 \mathrm{H}), 1.55-1.66(\mathrm{~m}, 3 \mathrm{H}), 1.52(\mathrm{~d}, J=$ $6.9 \mathrm{~Hz}, 3 \mathrm{H}), 1.47-1.36(\mathrm{~m}, 1 \mathrm{H}), 1.10-0.98(\mathrm{~m}, 1 \mathrm{H}), 0.76-0.82(\mathrm{~m}, 6 \mathrm{H})$ (Figure S12). ${ }^{13} \mathrm{C}$ NMR $(100$ MHz, DMSO-d 6 ) $\delta 174.2(\mathrm{C}=\mathrm{O}$ amide $), 140.6,133.8,130.9,129.1,127.6,126.6,126.1,125.8,123.7$, 122.9, 59.7, 44.1, 39.06, 24.0, 22.0, 16.4, 11.9 (Figure S15).

(2R,3S)-2-amino- $N$-[4-(tert-butyl)benzyl]-3-methylpentanamide $(3 \mathrm{~b}):(1.56 \mathrm{~g}, \% 94)$. Mp: $70-71{ }^{\circ} \mathrm{C}$. Anal. Calcd (\%) for $\mathrm{C}_{17} \mathrm{H}_{28} \mathrm{~N}_{2} \mathrm{O}$ : C, 73.91; H, 10.14. Found: C, $73.85 \mathrm{H}, 10.19$. IR: $\left(\mathrm{v} / \mathrm{cm}^{-1}\right) 3396,3331$, $3263,3063,2953,2928,2903,2869,1667,1633,1540,1510,1462,1443,1409,1375,1362,1300$, $1267,1244,1228,1183,1155,1135,1115,1107,1049,1021,945,928,891,825$ (Figure S16). ${ }^{1} \mathrm{H}$ NMR $\left(400 \mathrm{MHz}, \mathrm{DMSO}_{-} \mathrm{d}_{6}\right) \delta 8.26(\mathrm{t}, J=5.8 \mathrm{~Hz}, 1 \mathrm{H}), 7.33(\mathrm{~d}, J=8.3 \mathrm{~Hz}, 2 \mathrm{H}), 7.19(\mathrm{~d}, J=8.3 \mathrm{~Hz}, 2 \mathrm{H}), 4.26$ $(\mathrm{t}, J=6.2 \mathrm{~Hz}, 2 \mathrm{H}), 3.02(\mathrm{~d}, J=5.6 \mathrm{~Hz}, 1 \mathrm{H}), 1.66-1.59(\mathrm{~m}, 3 \mathrm{H}), 1.49-1.43(\mathrm{~m}, 1 \mathrm{H}), 1.27(\mathrm{~s}, 9 \mathrm{H}), 1.12$ - $1.05(\mathrm{~m}, 1 \mathrm{H}), 0.84(\mathrm{~m}, 6 \mathrm{H})$ (Figure S17). ${ }^{13} \mathrm{C}$ NMR (100 MHz, DMSO-d 6 ) $\delta 175.1$ (C=O amide), 149.5, 137.1, 127.6, 125.4, 59.9, 42.1, 38.96, 34.59, 31.6, 24.2, 16.4, 12.0 (Figure S18).

(2R,3S)-2-amino-N-(4-butylbenzyl)-3-methylpentanamide (3c): orange viscos (1.41 g, \%85). Anal. Calcd (\%) for $\mathrm{C}_{16} \mathrm{H}_{26} \mathrm{~N}_{2} \mathrm{O}$ : C, 73.28; H, 9.92. Found: C, $73.35 \mathrm{H}, 9.85$. IR: (v/cm $\left.{ }^{-1}\right) 3294,2959,2928$, 2872, 2853, 1658, 1602, 1589, 1515, 1455, 1409, 1378, 1309, 1244, 1200, 1174, 1132, 1118, 828 (Figure S19). ${ }^{1} \mathrm{H}$ NMR (400 MHz, $\left.\mathrm{CDCl}_{3}\right) \delta 9.50(\mathrm{~s}, 1 \mathrm{H}), 7.49(\mathrm{~d}, J=8.5 \mathrm{~Hz}, 2 \mathrm{H}), 7.10(\mathrm{~d}, J=8.5 \mathrm{~Hz}, 2 \mathrm{H})$, $3.34(\mathrm{~d}, J=3.8 \mathrm{~Hz}, 1 \mathrm{H}), 2.55(\mathrm{~m}, 2 \mathrm{H}), 1.60-1.52(\mathrm{~m}, 4 \mathrm{H}), 1.47-1.38(\mathrm{~m}, 2 \mathrm{H}), 1.36-1.26(\mathrm{~m}, 3 \mathrm{H})$, $1.16-1.10(\mathrm{~m}, 2 \mathrm{H}), 0.96(\mathrm{~d}, \mathrm{~J}=6.9 \mathrm{~Hz}, 3 \mathrm{H}), 0.91(\mathrm{~m}, 6 \mathrm{H})$ (Figure S20). ${ }^{13} \mathrm{C} \mathrm{NMR}\left(100 \mathrm{MHz}, \mathrm{CDCl}_{3}\right)$ $\delta 172.8(\mathrm{C}=\mathrm{O}$ amide $), 138.6,135.5,128.4,119.6,60.2,38.1,35.0,33.7,23.7,22.2(2 \mathrm{C}), 16.3,13.9,11.9$ (Figure S21).

\subsection{General Synthesis of Oxopentan-Phthalamide Derivative 4 (a-c)}

The compounds 3a-c $(5.0 \mathrm{mmol})$, THF $(30 \mathrm{~mL})$, pyridine $(5.0 \mathrm{mmol})$ were added in two necked flask at $0{ }^{\circ} \mathrm{C}$. A drying tube consisting of $\mathrm{CaCl}_{2}$ was inserted to the one necked of the flask. A solution of phthaloyl dichloride $(2.5 \mathrm{mmol})$ in THF $(30 \mathrm{~mL})$ was added to the reaction flask via dropping funnel for one hour. A white precipitate was formed while addition of phthaloyl dichloride. The reaction mixture was stirred overnight at room temperature. After the completion of reaction by TLC check, the precipitated solid was filtered. The solvent was removed under reduced pressure and chloroform (100 $\mathrm{mL}$ ) was added to the reaction flask, extracted with $1 \mathrm{M} \mathrm{HCl}, 1 \mathrm{M} \mathrm{KOH}$ and distilled water sequentially, dried $\left(\mathrm{MgSO}_{4}\right)$, filtered, and the solvent was removed under reduced pressure to yield the product. 
Erenler et al., Org. Commun. (2021) 14:3 294-299

Synthesis of N1-[(2S,3S)-3-methyl-1-\{[(R)-1-(naphthalen-1-yl)ethyl]amino\}-1-oxopentan-2-yl]-N2[(2R,3S)-3-methyl-1-\{[(S)-1-(naphthalen-1-yl)ethyl]amino\}-1-oxopentan-2-yl]phthalamide (4a): (3.32 g, 95\%). Mp: 304-305 ${ }^{\circ}$ C. Anal. Calcd (\%) for $\mathrm{C}_{44} \mathrm{H}_{50} \mathrm{~N}_{4} \mathrm{O}_{4}$ : C, 75.62; H, 7.21. Found: C, 75.58 H, 7.16. IR: $\left(\mathrm{v} / \mathrm{cm}^{-1}\right) 3272,3054,2965,2931,2875,1630,1524,1484,1455,1381,1370,1350,1317,1286$, 1250, 1216, 1172, 1146, 1107, 1087, 1001, 919, 775 (Figure S22). ${ }^{1} \mathrm{H}$ NMR (400 MHz, DMSO-d 6 ) $\delta$ $8.86(\mathrm{~m}, 4 \mathrm{H}), 8.55(\mathrm{~d}, J=8.8 \mathrm{~Hz}, 2 \mathrm{H}), 8.41-8.37(\mathrm{~m}, 2 \mathrm{H}), 8.16(\mathrm{~d}, J=7.6 \mathrm{~Hz}, 2 \mathrm{H}), 8.04(\mathrm{dd}, J=7.7$, $1.5 \mathrm{~Hz}, 2 \mathrm{H}), 7.90(\mathrm{~m}, 2 \mathrm{H}), 7.81(\mathrm{~d}, J=8.1 \mathrm{~Hz}, 2 \mathrm{H}), 7.60(\mathrm{~d}, J=7.0 \mathrm{~Hz}, 2 \mathrm{H}), 7.57-7.48(\mathrm{~m}, 2 \mathrm{H}), 7.45$ $(\mathrm{t}, J=7.7 \mathrm{~Hz}, 2 \mathrm{H}), 5.77(\mathrm{t}, J=8.8 \mathrm{~Hz}, 2 \mathrm{H}), 4.47(\mathrm{t}, J=8.8 \mathrm{~Hz}, 2 \mathrm{H}), 1.94(\mathrm{~m}, 2 \mathrm{H}), 1.52(\mathrm{~d}, J=6.9 \mathrm{~Hz}$, $6 \mathrm{H}), 1.49(\mathrm{~m}, 2 \mathrm{H}), 1.20-1.13(\mathrm{~m}, 2 \mathrm{H}), 0.83-0.78(\mathrm{~m}, 12 \mathrm{H})$ (Figure S23). ${ }^{13} \mathrm{C}$ NMR (100 MHz, DMSO$\left.\mathrm{d}_{6}\right) \delta 170.7(\mathrm{C}=\mathrm{O}$ pentanamide $), 166.4(\mathrm{C}=\mathrm{O}$ phthalimide $), 144.5,140.9,134.9,133.8,130.7,129.1$, 127.2, 126.8, 126.5, 126.0, 125.8, 123.6, 123.1, 58.5, 44.6, 36.7, 25.2, 22.4, 15.9, 11.1 (Figure S24).

Synthesis of N1-[(2R,3S)-1-\{[4-(tert-butyl)benzyl]amino\}-3-methyl-1-oxopentan-2-yl]-N2-[(2S,3S)-1\{[4-(tert-butyl)benzyl]amino\}-3-methyl-1-oxopentan-2-yl]phthalamide (4b): $(3.11 \mathrm{~g}, 91 \%)$. Mp: 247$249{ }^{\circ} \mathrm{C}$. Anal. Calcd (\%) for $\mathrm{C}_{42} \mathrm{H}_{58} \mathrm{~N}_{4} \mathrm{O}_{4}$ : C, 73.87; H, 8.56. Found: C, $73.91 \mathrm{H}, 8.61$. IR: $\left(\mathrm{v} / \mathrm{cm}^{-1}\right) 3275$, 3060, 2959, 2875, 1636, 1524, 1459, 1381, 1362, 1342, 1289, 1269, 1250, 1219, 1172, 1152, 1107, 1057, 1021, 928, 808 (Figure S26). ${ }^{1} \mathrm{H}$ NMR (400 MHz, DMSO-d 6 ) $\delta 8.53$ (t, $J=5.8 \mathrm{~Hz}, 2 \mathrm{H}$ ), 8.48 (d, $J=8.7 \mathrm{~Hz}, 2 \mathrm{H}), 8.32(\mathrm{brs}, 2 \mathrm{H}), 8.02(\mathrm{dd}, J=7.7,1.6 \mathrm{~Hz}, 2 \mathrm{H}), 7.32(\mathrm{~d}, J=8.3 \mathrm{~Hz}, 4 \mathrm{H}), 7.19(\mathrm{~d}, J=8.2$ $\mathrm{Hz}, 4 \mathrm{H}), 4.41(\mathrm{t}, J=8.6 \mathrm{~Hz}, 2 \mathrm{H}), 4.27(\mathrm{dd}, J=5.2,3.1 \mathrm{~Hz}, 4 \mathrm{H}), 1.96(\mathrm{~d}, J=6.6 \mathrm{~Hz}, 2 \mathrm{H}), 1.50-1.56$ $(\mathrm{m}, 2 \mathrm{H}), 1.25(\mathrm{~s}, 18 \mathrm{H}), 1.19(\mathrm{~m}, 2 \mathrm{H}), 0.9(\mathrm{~d}, J=6.8 \mathrm{~Hz}, 6 \mathrm{H}), 0.85(\mathrm{t}, J=7.4 \mathrm{~Hz}, 6 \mathrm{H})$ (Figure S27). ${ }^{13} \mathrm{C}$ NMR (100 MHz, DMSO-d 6 ) $\delta 171.5,166.5,149.6,136.7,134.9,130.7,127.5$ (2C overlapped), 125.4, 58.5, 42.3, 36.4, 34.6, 31.6, 25.3, 16.0, 11.2 (Figure S30).

N1-[(2R,3S)-1-\{[4-butylbenzyl]amino\}-3-methyl-1-oxopentan-2-yl]-N2-[(2S,3S)-1-\{[4-

butylbenzyl]amino\}-3-methyl-1-oxopentan-2-yl]phthalamide (4c): $(3.24 \mathrm{~g}, 95 \%)$. Mp: 140-142 ${ }^{\circ} \mathrm{C}$. Anal. Calcd (\%) for $\mathrm{C}_{42} \mathrm{H}_{58} \mathrm{~N}_{4} \mathrm{O}_{4}$ : C, 73.87; H, 8.56. Found: C, 73.91 H, 8.61. IR: (v/cm $\left.{ }^{-1}\right) 3264,2959$, 2931, 2875, 2856, 2601, 2495, 1639, 1611, 1524, 1474, 1415, 1398, 1373, 1317, 1281, 1250, 1174, 1074, 1037 (Figure S31). ${ }^{1} \mathrm{H}$ NMR (400 MHz, DMSO-d $)$ ) $\delta 10.12(\mathrm{~s}, 2 \mathrm{H}), 8.65(\mathrm{~d}, J=7.9 \mathrm{~Hz}, 2 \mathrm{H}), 8.04$ $(\mathrm{dd}, J=7.7,1.5 \mathrm{~Hz}, 2 \mathrm{H}), 7.54(\mathrm{~d}, \mathrm{~J}=8.4 \mathrm{~Hz}, 4 \mathrm{H}), 7.12(\mathrm{~d}, J=8.4 \mathrm{~Hz}, 4 \mathrm{H}), 4.49(\mathrm{t}, J=8.7 \mathrm{~Hz}, 2 \mathrm{H})$, $2.51(\mathrm{~m}, 10 \mathrm{H}), 2.03(\mathrm{~d}, J=6.5 \mathrm{~Hz}, 2 \mathrm{H}), 1.63-1.48(\mathrm{~m}, 6 \mathrm{H}), 1.33-1.23(\mathrm{~m}, 6 \mathrm{H}), 0.94(\mathrm{~d}, J=6.7 \mathrm{~Hz}$, $6 \mathrm{H}), 0.89\left(\mathrm{t}, J=7.3 \mathrm{~Hz}, 12 \mathrm{H}\right.$ ) (Figure S32). ${ }^{13} \mathrm{C}$ NMR (101 MHz, DMSO-d 6 ) $\delta 170.7,166.7,137.8$, 137.0, 134.8, 130.8, 128.9, 128.6, 119.9, 59.2, 36.4, 34.7, 33.7, 25.5, 22.1 (2 peaks overlapped), 15.8, 14.2, 11.1 (Figure S33).

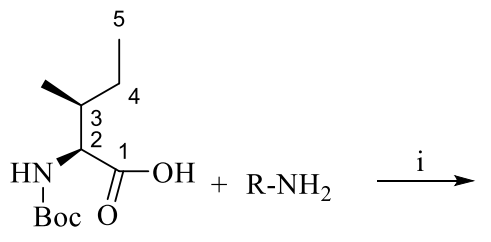

1

a)<smiles>[R]C(C)c1cccc2ccccc12</smiles>

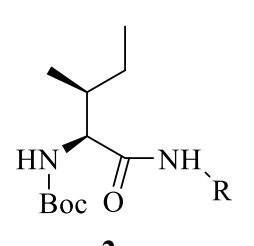

2

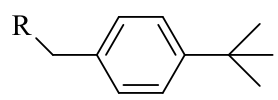

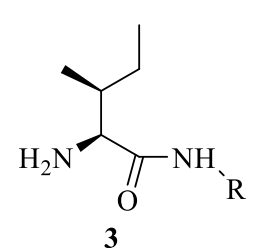

c)

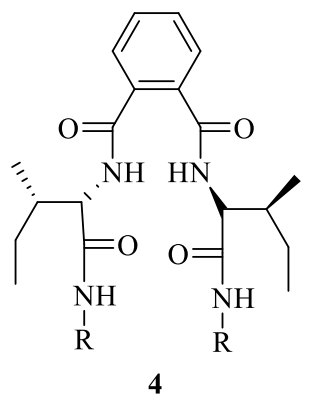

4

Scheme 1. Reagent and conditions: i) R-NH $\mathrm{NH}_{2} \mathrm{OH}, \mathrm{HOBt}, \mathrm{DCC}, \mathrm{CH}_{2} \mathrm{Cl}_{2}$, rt, overnight, 2a $91 \%, \mathbf{2 b} 74 \%$, 2c $90 \%$; ii) $\mathrm{AcOH}, \mathrm{TFA}, \mathrm{CH}_{2} \mathrm{Cl}_{2}, \mathrm{rt}$, overnight, 3a 95\%, 3b 94\%, 3c 85\%; iii) phthaloyl dichloride, THF, rt, overnight, 4a 95\%, 4b 91\%, 4c 95\%. 
Preparation of carboxyl and amino groups

\section{Results and Discussion}

The synthetic pathways for the synthesised compounds were given in Scheme 1. Firstly, the reaction of tert-butoxycarbonyl-L-isoleucine $\mathbf{1}$ with aromatic amine (a-c) in the presence of HOBt and DCC in $\mathrm{CH}_{2} \mathrm{Cl}_{2}$ at room temperature yielded the formation of compound 2 (a-c). 1-hydroxy-1Hbenzotriazole ( $\mathrm{HOBt}$ ) was used as a coupling reagent to reduce the epimerisation level and to increase the reaction yield ${ }^{16}$.

Removal of Boc protection group of $\mathbf{2}(\mathbf{a}-\mathbf{c})$ with TFA gave the compound $\mathbf{3}(\mathbf{a}-\mathbf{c})$. The coupling reaction was executed for the last stage. In this stage, the compound $\mathbf{3}$ (a-c) was treated with phthaloyl dichloride in THF yielded the formation of compound $\mathbf{4}$ (a-c).

As a conclusion, starting from $\mathrm{N}-\mathrm{Boc}$ protected isoleucine, three $\mathrm{N}-\mathrm{Boc}$ protected amide derivatives (2a-c), monoamide derivatives (3a-c) and bisamide derivatives (4a-c) were synthesized with good yields. The desired products (4a-c) and intermediate products could be valuable compounds for pharmaceutical materials. Since similar scaffold compounds have been used in drug production.

\section{Acknowledgements}

This research was supported by Batman University, Scientific Research Council (BTUBAP2018-FED-2).

\section{Supporting Information}

Supporting information accompanies this paper on http://www.acgpubs.org/journal/organiccommunications

\section{ORCID}

Murat Sunkur: 0000-0002-8513-7860

Sara Aydin: 0000-0002-5067-9467

Tarık Aral: 0000-0002-6612-2751

Beşir Dağ: 0000-0002-5208-3552

Ramazan Erenler: 0000-0002-0505-3190

\section{References}

[1] Mohammadi, G.Z.; Moradi, R.; Ahmadi, T.; Gholamzadeh, P. The molecular diversity scope of 4hydroxycoumarin in the synthesis of heterocyclic compounds via multicomponent reactions. Mol. diver. 2019, 23 (4), 1029-1064.

[2] Kumar, P.; Sangam; Ahmad, M.I. A brief review on recent advancements and biological activities of aryl propionic acid derivatives. Int. J. Pharm. Sci. Res. 2020, 11 (9), 4180-4188.

[3] Ogasawara, Y. New enzymes for peptide biosynthesis in microorganisms. Biosci. Biotechnol. Biochem. 2019, 83 (4), 589-597.

[4] Tretyakova, E.V.; Smirnova, I.E.; Salimova, E.V.; Odinokov, V.N. Synthesis and antiviral activity of maleopimaric and quinopimaric acids' derivatives. Bioorgan. Med. Chem. 2015, 23 (20), 6543-6550.

[5] Amini, A.; Raheem, S.; Steiner, A.; Deeba, F.; Ahmad, Z. Insect venom peptides as potent inhibitors of Escherichia coli ATP synthase. Int. J. Biol. Macromol. 2020, 150, 23-30.

[6] Naidu, K.M.; Gajanan, R.N.; Sekhar, K. Design, synthesis and biological evaluation of 5-(2-(4(substituted benzo d isoxazol-3-yl) piperazin-1-yl)acetyl)indolin-2-one and 5-(2-(4-substitutedpiperazin1-yl)acetyl)indolin-2-one analogues as novel anti-tubercular agents. Arab. J. Chem. 2019, 12 (8), 2418 2429.

[7] Ding, C.R.; Pan, Y.Y.; Tan, C.X. Synthesis and biological activity of aryl thiazole piperidine amide compounds. Chinese J. Org. Chem. 2020, 40 (2), 528-535. 
Erenler et al., Org. Commun. (2021) 14:3 294-299

[8] Tsikolia, M.; Bernier, U.R.; Agramonte, N.M.; Estep, A.S.; Becnel, J.J.; Linthicum, K.J.; Bloomquist, J.R. Insecticidal and repellent properties of novel trifluoromethylphenyl amides III. Pest. Biochem. Physiol. 2019, 161, 5-11.

[9] Yamaguchi-Sasaki, T.; Tamura, Y.; Ogata, Y.; Kawaguchi, T.; Kurosaka, J.; Sugaya, Y.; Iwakiri, K.; Busujima, T.; Takahashi, R.; Ueda-Yonemoto, N.; Tanigawa, E.; Abe-Kumasaka, T.; Sugiyama, H.; Kanuma, K. Design and synthesis of 2-(1-Alkylaminoalkyl)pyrazolo 1,5-a pyrimidines as new respiratory syncytial virus fusion protein inhibitors. Chem. Pharm. Bull. 2020, 68 (4), 345-362.

[10] Graul, A.; Castaner, J. Atorvastatin calcium. Drugs Future 1997, 22 (9), 956-968.

[11] Patchett, A.A. Excursions in drug discovery. J. Med. Chem. 1993, 36 (15), 2051-2058.

[12] de Gasparo, M.; Whitebread, S. Binding of valsartan to mammalian angiotensin AT1 receptors. Regul. Pept. 1995, 59 (3), 303-311.

[13] Ananthanarayanan, V.; Tetreault, S.; Saint-Jean, A. Interaction of calcium channel antagonists with calcium: spectroscopic and modeling studies on diltiazem and its $\mathrm{Ca}^{2+}$ complex. J. Med. Chem. 1993, 36 (10), 1324-1332.

[14] Küçükbay, H.; Buğday, N.; Küçükbay, F.Z.; Berrino, E.; Bartolucci, G.; Del Prete, S.; Capasso, C.; Supuran, C.T. Synthesis and carbonic anhydrase inhibitory properties of novel 4-(2-aminoethyl) benzenesulfonamide-dipeptide conjugates. Bioorg. Chem. 2019, 83, 414-423.

[15] Küçükbay, H.; Gönül, Z.; Küçükbay, F.Z.; Tekin, Z.; Angeli, A.; Bartolucci, G.; Supuran, C.T.; Tatlıc1, E.; Apohan, E.; Yeşilada, Ö. Synthesis of new 7-amino-3, 4-dihydroquinolin-2 (1H)-one-peptide derivatives and their carbonic anhydrase enzyme inhibition, antioxidant, and cytotoxic activities. Arch. Pharm. (Weinheim) 2021, e2100122.

[16] Valeur, E.; Bradley, M. Amide bond formation: beyond the myth of coupling reagents. Chem. Soc. Rev. 2009, 38 (2), 606-631.

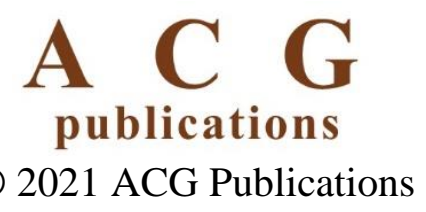

